Supplement of Hydrol. Earth Syst. Sci., 21, 635-650, 2017

http://www.hydrol-earth-syst-sci.net/21/635/2017/

doi:10.5194/hess-21-635-2017-supplement

(C) Author(s) 2017. CC Attribution 3.0 License.

(c) (1)

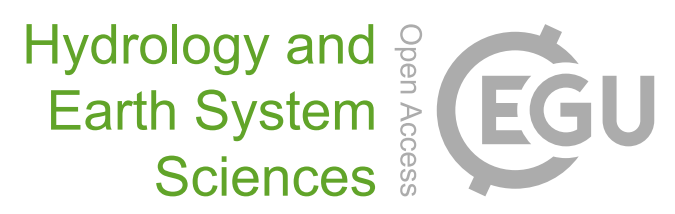

Supplement of

\title{
Evaluation of snow data assimilation using the ensemble Kalman filter for seasonal streamflow prediction in the western United States
}

Chengcheng Huang et al.

Correspondence to: Andrew J. Newman (anewman@ucar.edu)

The copyright of individual parts of the supplement might differ from the CC-BY 3.0 licence. 
Table S.1 Evaluation statistics for percentile interpolation before and after DA for 3month window.

\begin{tabular}{|c|c|c|c|c|c|c|}
\hline Basin name & $\begin{array}{c}\text { Forecast error } \\
\text { variance }(\mathrm{P})\end{array}$ & R-RMSE & $\mathrm{R}$ & NSE & Bias & CRPS \\
\hline \multirow{4}{*}{ Crystal River } & No DA & 0.124 & 0.93 & 0.83 & -16.6 & 34.9 \\
\hline & $\mathrm{P}$ & 0.118 & 0.94 & 0.85 & -12.3 & 33.1 \\
\hline & $1 / 2 \cdot \mathrm{P}$ & 0.120 & 0.94 & 0.84 & -14.3 & 33.4 \\
\hline & $2 \cdot \mathrm{P}$ & 0.115 & 0.94 & 0.86 & -9.2 & 32.7 \\
\hline \multirow{4}{*}{$\begin{array}{l}\text { Vallecito } \\
\text { River }\end{array}$} & No DA & 0.110 & 0.97 & 0.87 & -33.9 & 30 \\
\hline & $\mathrm{P}$ & 0.103 & 0.98 & 0.89 & -30.7 & 28.4 \\
\hline & $1 / 2 \cdot \mathrm{P}$ & 0.106 & 0.97 & 0.89 & -32.1 & 28.9 \\
\hline & $2 \cdot \mathrm{P}$ & 0.099 & 0.98 & 0.90 & -28.3 & 27.9 \\
\hline \multirow{4}{*}{ Greys River } & No DA & 0.113 & 0.95 & 0.89 & -3.0 & 18.7 \\
\hline & $\mathrm{P}$ & 0.082 & 0.97 & 0.94 & -3.9 & 15.6 \\
\hline & $1 / 2 \cdot \mathrm{P}$ & 0.09 & 0.97 & 0.93 & -3.8 & 16.3 \\
\hline & $2 \cdot \mathrm{P}$ & 0.077 & 0.98 & 0.95 & -3.8 & 15.1 \\
\hline \multirow{4}{*}{ SF Tolt River } & No DA & 0.242 & 0.76 & 0.47 & 81.6 & 177 \\
\hline & $\mathrm{P}$ & 0.234 & 0.82 & 0.51 & 120.0 & 156.9 \\
\hline & $1 / 2 \cdot \mathrm{P}$ & 0.234 & 0.81 & 0.50 & 111.6 & 164.7 \\
\hline & $2 \cdot \mathrm{P}$ & 0.234 & 0.84 & 0.50 & 132.8 & 150.6 \\
\hline \multirow{4}{*}{$\begin{array}{l}\text { SF Payette } \\
\text { River }\end{array}$} & No DA & 0.124 & 0.96 & 0.90 & -26.8 & 29.4 \\
\hline & $\mathrm{P}$ & 0.118 & 0.97 & 0.91 & -24.9 & 28.8 \\
\hline & $1 / 2 \cdot \mathrm{P}$ & 0.117 & 0.967 & 0.91 & -25.9 & 28.1 \\
\hline & $2 \cdot \mathrm{P}$ & 0.119 & 0.96 & 0.90 & -23.4 & 30 \\
\hline \multirow{4}{*}{ Smith River } & No DA & 0.211 & 0.87 & 0.72 & -28.5 & 76.4 \\
\hline & $\mathrm{P}$ & 0.178 & 0.91 & 0.80 & -26.3 & 65.9 \\
\hline & $1 / 2 \cdot P$ & 0.187 & 0.90 & 0.78 & -28.0 & 69.2 \\
\hline & $2 \cdot \mathrm{P}$ & 0.168 & 0.92 & 0.82 & -22.9 & 62.1 \\
\hline \multirow{4}{*}{$\begin{array}{l}\text { General } \\
\text { Creek }\end{array}$} & No DA & 0.118 & 0.98 & 0.95 & -15.5 & 36.6 \\
\hline & $\mathrm{P}$ & 0.092 & 0.99 & 0.97 & -17.4 & 29.8 \\
\hline & $1 / 2 \cdot \mathrm{P}$ & 0.097 & 0.99 & 0.97 & -18.2 & 30.3 \\
\hline & $2 \cdot \mathrm{P}$ & 0.089 & 0.99 & 0.97 & -15.2 & 30.9 \\
\hline \multirow{4}{*}{$\begin{array}{c}\text { Blackwood } \\
\text { Creek }\end{array}$} & No DA & 0.127 & 0.98 & 0.94 & -52.4 & 55.1 \\
\hline & $\mathrm{P}$ & 0.118 & 0.98 & 0.94 & -50.0 & 52.8 \\
\hline & $1 / 2 \cdot \mathrm{P}$ & 0.119 & 0.98 & 0.94 & -53.2 & 51.1 \\
\hline & $2 \cdot \mathrm{P}$ & 0.117 & 0.98 & 0.95 & -44.5 & 55 \\
\hline \multirow{4}{*}{ Merced River } & No DA & 0.266 & 0.95 & 0.74 & -105.4 & 83.4 \\
\hline & $\mathrm{P}$ & 0.253 & 0.96 & 0.76 & -106.0 & 85 \\
\hline & $1 / 2 \cdot \mathrm{P}$ & 0.258 & 0.96 & 0.75 & -107.8 & 86.6 \\
\hline & $2 \cdot \mathrm{P}$ & 0.245 & 0.96 & 0.78 & -102.6 & 81.9 \\
\hline
\end{tabular}


Table S.2 Evaluation statistics for Z-score interpolation before and after DA for 3month window.

\begin{tabular}{|c|c|c|c|c|c|c|}
\hline Basin name & $\begin{array}{c}\text { Forecast error } \\
\text { variance }(\mathrm{P})\end{array}$ & R-RMSE & $\mathrm{R}$ & NSE & Bias & CRPS \\
\hline \multirow{4}{*}{ Crystal River } & No DA & 0.124 & 0.93 & 0.83 & -16.6 & 34.9 \\
\hline & $\mathrm{P}$ & 0.119 & 0.94 & 0.85 & -11.2 & 33.9 \\
\hline & $1 / 2 \cdot \mathrm{P}$ & 0.12 & 0.94 & 0.84 & -13.4 & 34.1 \\
\hline & $2 \cdot \mathrm{P}$ & 0.117 & 0.95 & 0.85 & -8.6 & 33.6 \\
\hline \multirow{4}{*}{$\begin{array}{c}\text { Vallecito } \\
\text { River }\end{array}$} & No DA & 0.11 & 0.97 & 0.87 & -33.9 & 30 \\
\hline & $\mathrm{P}$ & 0.1 & 0.98 & 0.9 & -29.8 & 28.7 \\
\hline & $1 / 2 \cdot \mathrm{P}$ & 0.103 & 0.98 & 0.89 & -31.2 & 29.1 \\
\hline & $2 \cdot \mathrm{P}$ & 0.098 & 0.97 & 0.9 & -28.3 & 28.2 \\
\hline \multirow{4}{*}{ Greys River } & No DA & 0.113 & 0.95 & 0.89 & -3 & 18.7 \\
\hline & $\mathrm{P}$ & 0.092 & 0.97 & 0.93 & -3.5 & 16.2 \\
\hline & $1 / 2 \cdot \mathrm{P}$ & 0.098 & 0.97 & 0.92 & -3.4 & 16.8 \\
\hline & $2 \cdot \mathrm{P}$ & 0.087 & 0.97 & 0.93 & -3.5 & 16 \\
\hline \multirow{4}{*}{ SF Tolt River } & No DA & 0.242 & 0.76 & 0.47 & 81.6 & 177 \\
\hline & $\mathrm{P}$ & 0.182 & 0.88 & 0.7 & 102.6 & 127.9 \\
\hline & $1 / 2 \cdot \mathrm{P}$ & 0.193 & 0.86 & 0.66 & 97.2 & 137.3 \\
\hline & $2 \cdot \mathrm{P}$ & 0.174 & 0.9 & 0.73 & 109.5 & 121.3 \\
\hline \multirow{4}{*}{$\begin{array}{l}\text { SF Payette } \\
\text { River }\end{array}$} & No DA & 0.124 & 0.96 & 0.9 & -26.8 & 29.4 \\
\hline & $\mathrm{P}$ & 0.117 & 0.97 & 0.91 & -22.7 & 28.7 \\
\hline & $1 / 2 \cdot \mathrm{P}$ & 0.115 & 0.97 & 0.91 & -23 & 27.9 \\
\hline & $2 \cdot \mathrm{P}$ & 0.122 & 0.96 & 0.9 & -23.4 & 30 \\
\hline \multirow{4}{*}{ Smith River } & No DA & 0.211 & 0.87 & 0.72 & -28.5 & 76.4 \\
\hline & $\mathrm{P}$ & 0.173 & 0.91 & 0.81 & -30.6 & 63.7 \\
\hline & $1 / 2 \cdot \mathrm{P}$ & 0.183 & 0.9 & 0.79 & -31.2 & 67.6 \\
\hline & $2 \cdot \mathrm{P}$ & 0.163 & 0.92 & 0.84 & -29.5 & 60 \\
\hline \multirow{4}{*}{$\begin{array}{c}\text { General } \\
\text { Creek }\end{array}$} & No DA & 0.118 & 0.98 & 0.95 & -15.5 & 36.6 \\
\hline & $P$ & 0.096 & 0.99 & 0.97 & -20 & 30 \\
\hline & $1 / 2 \cdot \mathrm{P}$ & 0.10 & 0.99 & 0.97 & -18.7 & 31 \\
\hline & $2 \cdot \mathrm{P}$ & 0.096 & 0.99 & 0.97 & -21.2 & 30.4 \\
\hline \multirow{4}{*}{$\begin{array}{c}\text { Blackwood } \\
\text { Creek }\end{array}$} & No DA & 0.127 & 0.98 & 0.94 & -52.4 & 55.1 \\
\hline & $\mathrm{P}$ & 0.118 & 0.98 & 0.94 & -53.5 & 51.8 \\
\hline & $1 / 2 \cdot \mathrm{P}$ & 0.118 & 0.98 & 0.94 & -53.2 & 50.6 \\
\hline & $2 \cdot \mathrm{P}$ & 0.120 & 0.98 & 0.94 & -53.5 & 53.7 \\
\hline \multirow{4}{*}{ Merced River } & No DA & 0.266 & 0.95 & 0.74 & -105.4 & 83.4 \\
\hline & $\mathrm{P}$ & 0.24 & 0.97 & 0.79 & -99.3 & 79.8 \\
\hline & $1 / 2 \cdot \mathrm{P}$ & 0.246 & 0.96 & 0.78 & -101.4 & 83.1 \\
\hline & $2 \cdot \mathrm{P}$ & 0.234 & 0.97 & 0.8 & -96.9 & 76 \\
\hline
\end{tabular}


Table S.3 Evaluation statistics for percentile interpolation before and after DA for 7day window.

\begin{tabular}{|c|c|c|c|c|c|c|}
\hline Basin name & $\begin{array}{c}\text { Forecast error } \\
\text { variance }(\mathrm{P})\end{array}$ & R-RMSE & $\mathrm{R}$ & NSE & Bias & CRPS \\
\hline \multirow{4}{*}{ Crystal River } & No DA & 0.124 & 0.93 & 0.83 & -16.6 & 34.9 \\
\hline & $\mathrm{P}$ & 0.117 & 0.95 & 0.85 & -14.5 & 32.8 \\
\hline & $1 / 2 \cdot \mathrm{P}$ & 0.119 & 0.94 & 0.85 & -15.7 & 33.2 \\
\hline & $2 \cdot \mathrm{P}$ & 0.115 & 0.95 & 0.86 & -12.4 & 32.7 \\
\hline \multirow{4}{*}{$\begin{array}{c}\text { Vallecito } \\
\text { River }\end{array}$} & No DA & 0.110 & 0.97 & 0.87 & -33.9 & 30 \\
\hline & $\mathrm{P}$ & 0.106 & 0.97 & 0.88 & -29.5 & 29.2 \\
\hline & $1 / 2 \cdot \mathrm{P}$ & 0.108 & 0.97 & 0.88 & -31.1 & 29.3 \\
\hline & $2 \cdot \mathrm{P}$ & 0.104 & 0.97 & 0.89 & -27.2 & 29.2 \\
\hline \multirow{4}{*}{ Greys River } & No DA & 0.113 & 0.95 & 0.89 & -3 & 18.7 \\
\hline & $\mathrm{P}$ & 0.098 & 0.97 & 0.92 & -0.3 & 16.2 \\
\hline & $1 / 2 \cdot \mathrm{P}$ & 0.104 & 0.96 & 0.91 & -1.4 & 17 \\
\hline & $2 \cdot \mathrm{P}$ & 0.092 & 0.97 & 0.93 & 1.2 & 15.6 \\
\hline \multirow{4}{*}{ SF Tolt River } & No DA & 0.242 & 0.76 & 0.47 & 81.6 & 177 \\
\hline & $\mathrm{P}$ & 0.183 & 0.86 & 0.7 & 83.2 & 128.9 \\
\hline & $1 / 2 \cdot \mathrm{P}$ & 0.196 & 0.84 & 0.65 & 77.1 & 137.3 \\
\hline & $2 \cdot \mathrm{P}$ & 0.182 & 0.88 & 0.7 & 96.4 & 128.9 \\
\hline \multirow{4}{*}{$\begin{array}{c}\text { SF Payette } \\
\text { River }\end{array}$} & No DA & 0.124 & 0.96 & 0.9 & -26.8 & 29.4 \\
\hline & $\mathrm{P}$ & 0.122 & 0.97 & 0.9 & -27.1 & 30.3 \\
\hline & $1 / 2 \cdot \mathrm{P}$ & 0.123 & 0.97 & 0.9 & -27.9 & 30.1 \\
\hline & $2 \cdot \mathrm{P}$ & 0.122 & 0.96 & 0.9 & -25.2 & 30.5 \\
\hline \multirow{4}{*}{ Smith River } & No DA & 0.211 & 0.87 & 0.72 & -28.5 & 76.4 \\
\hline & $\mathrm{P}$ & 0.178 & 0.91 & 0.8 & -25.8 & 66.3 \\
\hline & $1 / 2 \cdot \mathrm{P}$ & 0.189 & 0.9 & 0.78 & -27.1 & 70.1 \\
\hline & $2 \cdot \mathrm{P}$ & 0.166 & 0.92 & 0.83 & -23.5 & 61.8 \\
\hline \multirow{4}{*}{$\begin{array}{c}\text { General } \\
\text { Creek }\end{array}$} & No DA & 0.118 & 0.98 & 0.95 & -15.5 & 36.6 \\
\hline & $\mathrm{P}$ & 0.084 & 0.99 & 0.98 & -14.1 & 28.6 \\
\hline & $1 / 2 \cdot \mathrm{P}$ & 0.092 & 0.99 & 0.97 & -16.2 & 30 \\
\hline & $2 \cdot \mathrm{P}$ & 0.080 & 0.99 & 0.98 & -10.5 & 28.9 \\
\hline \multirow{4}{*}{$\begin{array}{l}\text { Blackwood } \\
\text { Creek }\end{array}$} & No DA & 0.127 & 0.98 & 0.94 & -52.4 & 55.1 \\
\hline & $\mathrm{P}$ & 0.108 & 0.99 & 0.95 & -52.3 & 48.2 \\
\hline & $1 / 2 \cdot \mathrm{P}$ & 0.112 & 0.99 & 0.95 & -54.5 & 48.6 \\
\hline & $2 \cdot \mathrm{P}$ & 0.105 & 0.99 & 0.96 & -48.5 & 49.1 \\
\hline \multirow{4}{*}{ Merced River } & No DA & 0.266 & 0.95 & 0.74 & -105.4 & 83.4 \\
\hline & $\mathrm{P}$ & 0.242 & 0.95 & 0.78 & -95.7 & 78.7 \\
\hline & $1 / 2 \cdot \mathrm{P}$ & 0.249 & 0.95 & 0.77 & -99.8 & 80.2 \\
\hline & $2 \cdot \mathrm{P}$ & 0.234 & 0.95 & 0.8 & -90.2 & 75.7 \\
\hline
\end{tabular}


Table S.4 Evaluation statistics for Z-score interpolation before and after DA for 7-day window.

\begin{tabular}{|c|c|c|c|c|c|c|}
\hline Basin name & $\begin{array}{c}\text { Forecast error } \\
\text { variance }(\mathrm{P})\end{array}$ & R-RMSE & $\mathrm{R}$ & NSE & Bias & CRPS \\
\hline \multirow{4}{*}{ Crystal River } & No DA & 0.124 & 0.93 & 0.83 & -16.6 & 34.9 \\
\hline & $\mathrm{P}$ & 0.122 & 0.94 & 0.84 & -17.9 & 34.6 \\
\hline & $1 / 2 \cdot \mathrm{P}$ & 0.122 & 0.94 & 0.84 & -18 & 34.6 \\
\hline & $2 \cdot \mathrm{P}$ & 0.121 & 0.94 & 0.84 & -17.4 & 34.4 \\
\hline \multirow{4}{*}{$\begin{array}{c}\text { Vallecito } \\
\text { River }\end{array}$} & No DA & 0.110 & 0.97 & 0.87 & -33.9 & 30 \\
\hline & $\mathrm{P}$ & 0.106 & 0.98 & 0.88 & -32.2 & 30.3 \\
\hline & $1 / 2 \cdot \mathrm{P}$ & 0.107 & 0.98 & 0.88 & -32.9 & 30.2 \\
\hline & $2 \cdot \mathrm{P}$ & 0.105 & 0.98 & 0.88 & -31.3 & 30.3 \\
\hline \multirow{4}{*}{ Greys River } & No DA & 0.113 & 0.95 & 0.89 & -3 & 18.7 \\
\hline & $\mathrm{P}$ & 0.097 & 0.97 & 0.92 & -2.6 & 16.4 \\
\hline & $1 / 2 \cdot \mathrm{P}$ & 0.102 & 0.96 & 0.91 & -2.8 & 16.9 \\
\hline & $2 \cdot \mathrm{P}$ & 0.093 & 0.97 & 0.92 & -2.3 & 16 \\
\hline \multirow{4}{*}{ SF Tolt River } & No DA & 0.242 & 0.76 & 0.47 & 81.6 & 177 \\
\hline & $\mathrm{P}$ & 0.178 & 0.9 & 0.71 & 109.8 & 124 \\
\hline & $1 / 2 \cdot \mathrm{P}$ & 0.191 & 0.88 & 0.67 & 108.4 & 134.2 \\
\hline & $2 \cdot \mathrm{P}$ & 0.170 & 0.91 & 0.74 & 112.7 & 116.8 \\
\hline \multirow{4}{*}{$\begin{array}{c}\text { SF Payette } \\
\text { River }\end{array}$} & No DA & 0.124 & 0.96 & 0.9 & -26.8 & 29.4 \\
\hline & $\mathrm{P}$ & 0.110 & 0.97 & 0.92 & -24.8 & 27 \\
\hline & $1 / 2 \cdot \mathrm{P}$ & 0.110 & 0.97 & 0.92 & -24.8 & 26.6 \\
\hline & $2 \cdot \mathrm{P}$ & 0.115 & 0.97 & 0.91 & -25.4 & 28.2 \\
\hline \multirow{4}{*}{ Smith River } & No DA & 0.211 & 0.87 & 0.72 & -28.5 & 76.4 \\
\hline & $\mathrm{P}$ & 0.169 & 0.92 & 0.82 & -34.2 & 62.4 \\
\hline & $1 / 2 \cdot \mathrm{P}$ & 0.177 & 0.91 & 0.81 & -33.2 & 66.1 \\
\hline & $2 \cdot \mathrm{P}$ & 0.161 & 0.93 & 0.84 & -34.9 & 59.3 \\
\hline \multirow{4}{*}{$\begin{array}{l}\text { General } \\
\text { Creek }\end{array}$} & No DA & 0.118 & 0.98 & 0.95 & -15.5 & 36.6 \\
\hline & $\mathrm{P}$ & 0.080 & 0.99 & 0.98 & -19.4 & 25.9 \\
\hline & $1 / 2 \cdot \mathrm{P}$ & 0.085 & 0.99 & 0.98 & -19 & 27 \\
\hline & $2 \cdot \mathrm{P}$ & 0.080 & 0.99 & 0.98 & -19.2 & 26.5 \\
\hline \multirow{4}{*}{$\begin{array}{l}\text { Blackwood } \\
\text { Creek }\end{array}$} & No DA & 0.127 & 0.98 & 0.94 & -52.4 & 55.1 \\
\hline & $\mathrm{P}$ & 0.114 & 0.99 & 0.95 & -59.1 & 51 \\
\hline & $1 / 2 \cdot \mathrm{P}$ & 0.114 & 0.99 & 0.95 & -58.1 & 49.7 \\
\hline & $2 \cdot \mathrm{P}$ & 0.116 & 0.99 & 0.95 & -59.7 & 52.3 \\
\hline \multirow{4}{*}{ Merced River } & No DA & 0.266 & 0.95 & 0.74 & -105.4 & 83.4 \\
\hline & $\mathrm{P}$ & 0.242 & 0.96 & 0.78 & -101.7 & 81.8 \\
\hline & $1 / 2 \cdot \mathrm{P}$ & 0.248 & 0.96 & 0.77 & -103 & 84.2 \\
\hline & $2 \cdot \mathrm{P}$ & 0.236 & 0.96 & 0.79 & -100.3 & 78.4 \\
\hline
\end{tabular}


Table S.5 Evaluation statistics for percentile interpolation before and after DA for 1year window.

\begin{tabular}{|c|c|c|c|c|c|c|}
\hline Basin name & $\begin{array}{c}\text { Forecast error } \\
\text { variance }(\mathrm{P})\end{array}$ & R-RMSE & $\mathrm{R}$ & NSE & Bias & CRPS \\
\hline \multirow{4}{*}{ Crystal River } & No DA & 0.124 & 0.93 & 0.83 & -16.6 & 34.9 \\
\hline & $\mathrm{P}$ & 0.118 & 0.94 & 0.85 & -11.2 & 33.1 \\
\hline & $1 / 2 \cdot \mathrm{P}$ & 0.120 & 0.94 & 0.84 & -13.6 & 33.6 \\
\hline & $2 \cdot \mathrm{P}$ & 0.115 & 0.95 & 0.86 & -7.7 & 32.5 \\
\hline \multirow{4}{*}{$\begin{array}{c}\text { Vallecito } \\
\text { River }\end{array}$} & No DA & 0.110 & 0.97 & 0.87 & -33.9 & 30 \\
\hline & $\mathrm{P}$ & 0.104 & 0.97 & 0.89 & -31.7 & 28.9 \\
\hline & $1 / 2 \cdot \mathrm{P}$ & 0.107 & 0.97 & 0.88 & -33.1 & 29.5 \\
\hline & $2 \cdot \mathrm{P}$ & 0.100 & 0.97 & 0.9 & -29.1 & 28.1 \\
\hline \multirow{4}{*}{ Greys River } & No DA & 0.113 & 0.95 & 0.89 & -3 & 18.7 \\
\hline & $\mathrm{P}$ & 0.090 & 0.98 & 0.93 & -16.8 & 17.4 \\
\hline & $1 / 2 \cdot \mathrm{P}$ & 0.089 & 0.97 & 0.93 & -13 & 16.9 \\
\hline & $2 \cdot \mathrm{P}$ & 0.094 & 0.98 & 0.92 & -20.4 & 18.4 \\
\hline \multirow{4}{*}{ SF Tolt River } & No DA & 0.242 & 0.76 & 0.47 & 81.6 & 177 \\
\hline & $\mathrm{P}$ & 0.223 & 0.84 & 0.55 & 119.6 & 158 \\
\hline & $1 / 2 \cdot \mathrm{P}$ & 0.221 & 0.83 & 0.56 & 104.1 & 162.2 \\
\hline & $2 \cdot \mathrm{P}$ & 0.228 & 0.86 & 0.53 & 141.7 & 152 \\
\hline \multirow{4}{*}{$\begin{array}{l}\text { SF Payette } \\
\text { River }\end{array}$} & No DA & 0.124 & 0.96 & 0.9 & -26.8 & 29.4 \\
\hline & $\mathrm{P}$ & 0.113 & 0.97 & 0.91 & -22 & 27.1 \\
\hline & $1 / 2 \cdot \mathrm{P}$ & 0.116 & 0.97 & 0.91 & -24.2 & 27.8 \\
\hline & $2 \cdot \mathrm{P}$ & 0.108 & 0.97 & 0.92 & -19 & 26.5 \\
\hline \multirow{4}{*}{ Smith River } & No DA & 0.211 & 0.87 & 0.72 & -28.5 & 76.4 \\
\hline & $\mathrm{P}$ & 0.179 & 0.91 & 0.8 & -26.1 & 67.5 \\
\hline & $1 / 2 \cdot \mathrm{P}$ & 0.187 & 0.9 & 0.78 & -29.4 & 70.3 \\
\hline & $2 \cdot \mathrm{P}$ & 0.170 & 0.91 & 0.82 & -20.4 & 63.6 \\
\hline \multirow{4}{*}{$\begin{array}{c}\text { General } \\
\text { Creek }\end{array}$} & No DA & 0.118 & 0.98 & 0.95 & -15.5 & 36.6 \\
\hline & $\mathrm{P}$ & 0.099 & 0.99 & 0.97 & -14 & 31.9 \\
\hline & $1 / 2 \cdot \mathrm{P}$ & 0.106 & 0.98 & 0.96 & -14.6 & 33.3 \\
\hline & $2 \cdot \mathrm{P}$ & 0.092 & 0.99 & 0.97 & -12.8 & 31.1 \\
\hline \multirow{4}{*}{$\begin{array}{c}\text { Blackwood } \\
\text { Creek }\end{array}$} & No DA & 0.127 & 0.98 & 0.94 & -52.4 & 55.1 \\
\hline & $\mathrm{P}$ & 0.118 & 0.98 & 0.94 & -47.1 & 50.8 \\
\hline & $1 / 2 \cdot \mathrm{P}$ & 0.121 & 0.98 & 0.94 & -49.2 & 51 \\
\hline & $2 \cdot \mathrm{P}$ & 0.117 & 0.98 & 0.95 & -43.9 & 52.7 \\
\hline \multirow{4}{*}{ Merced River } & No DA & 0.266 & 0.95 & 0.74 & -105.4 & 83.4 \\
\hline & $\mathrm{P}$ & 0.246 & 0.96 & 0.78 & -97.8 & 80.5 \\
\hline & $1 / 2 \cdot \mathrm{P}$ & 0.254 & 0.96 & 0.76 & -101.7 & 83.1 \\
\hline & $2 \cdot \mathrm{P}$ & 0.236 & 0.96 & 0.79 & -92.4 & 77.3 \\
\hline
\end{tabular}


Table S.6 Evaluation statistics for Z-score interpolation before and after DA for 1-year window.

\begin{tabular}{|c|c|c|c|c|c|c|}
\hline Basin name & $\begin{array}{c}\text { Forecast error } \\
\text { variance }(\mathrm{P})\end{array}$ & R-RMSE & $\mathrm{R}$ & NSE & Bias & CRPS \\
\hline \multirow{4}{*}{ Crystal River } & No DA & 0.124 & 0.93 & 0.83 & -16.6 & 34.9 \\
\hline & $\mathrm{P}$ & 0.115 & 0.94 & 0.86 & -7.2 & 32.4 \\
\hline & $1 / 2 \cdot \mathrm{P}$ & 0.118 & 0.94 & 0.85 & -10.8 & 33.2 \\
\hline & $2 \cdot \mathrm{P}$ & 0.111 & 0.94 & 0.87 & -2.4 & 31.7 \\
\hline \multirow{4}{*}{$\begin{array}{c}\text { Vallecito } \\
\text { River }\end{array}$} & No DA & 0.110 & 0.97 & 0.87 & -33.9 & 30 \\
\hline & $\mathrm{P}$ & 0.094 & 0.97 & 0.91 & -28.1 & 26.4 \\
\hline & $1 / 2 \cdot \mathrm{P}$ & 0.099 & 0.97 & 0.9 & -30.2 & 27.3 \\
\hline & $2 \cdot \mathrm{P}$ & 0.091 & 0.97 & 0.91 & -25.5 & 26 \\
\hline \multirow{4}{*}{ Greys River } & No DA & 0.113 & 0.95 & 0.89 & -3 & 18.7 \\
\hline & $\mathrm{P}$ & 0.106 & 0.96 & 0.9 & -13.6 & 19.2 \\
\hline & $1 / 2 \cdot \mathrm{P}$ & 0.107 & 0.96 & 0.9 & -10.3 & 18.5 \\
\hline & $2 \cdot \mathrm{P}$ & 0.106 & 0.97 & 0.9 & -17.1 & 20 \\
\hline \multirow{4}{*}{ SF Tolt River } & No DA & 0.242 & 0.76 & 0.47 & 81.6 & 177 \\
\hline & $\mathrm{P}$ & 0.182 & 0.87 & 0.7 & 93.8 & 128.4 \\
\hline & $1 / 2 \cdot P$ & 0.198 & 0.84 & 0.64 & 91.3 & 141.5 \\
\hline & $2 \cdot \mathrm{P}$ & 0.168 & 0.9 & 0.74 & 98.5 & 116.2 \\
\hline \multirow{4}{*}{$\begin{array}{c}\text { SF Payette } \\
\text { River }\end{array}$} & No DA & 0.124 & 0.96 & 0.9 & -26.8 & 29.4 \\
\hline & $\mathrm{P}$ & 0.114 & 0.97 & 0.91 & -23.5 & 27.8 \\
\hline & $1 / 2 \cdot P$ & 0.114 & 0.97 & 0.91 & -23.9 & 27.7 \\
\hline & $2 \cdot \mathrm{P}$ & 0.118 & 0.97 & 0.9 & -24.1 & 28.5 \\
\hline \multirow{4}{*}{ Smith River } & No DA & 0.211 & 0.87 & 0.72 & -28.5 & 76.4 \\
\hline & $\mathrm{P}$ & 0.179 & 0.9 & 0.8 & -26.8 & 65.6 \\
\hline & $1 / 2 \cdot \mathrm{P}$ & 0.189 & 0.89 & 0.78 & -28.1 & 69.3 \\
\hline & $2 \cdot \mathrm{P}$ & 0.167 & 0.91 & 0.83 & -24.6 & 61.3 \\
\hline \multirow{4}{*}{$\begin{array}{l}\text { General } \\
\text { Creek }\end{array}$} & No DA & 0.118 & 0.98 & 0.95 & -15.5 & 36.6 \\
\hline & $\mathrm{P}$ & 0.094 & 0.99 & 0.97 & -21.8 & 30.3 \\
\hline & $1 / 2 \cdot \mathrm{P}$ & 0.099 & 0.99 & 0.97 & -19.2 & 31.9 \\
\hline & $2 \cdot \mathrm{P}$ & 0.091 & 0.99 & 0.97 & -24.9 & 30.1 \\
\hline \multirow{4}{*}{$\begin{array}{l}\text { Blackwood } \\
\text { Creek }\end{array}$} & No DA & 0.127 & 0.98 & 0.94 & -52.4 & 55.1 \\
\hline & $\mathrm{P}$ & 0.112 & 0.99 & 0.95 & -54.1 & 50.4 \\
\hline & $1 / 2 \cdot P$ & 0.115 & 0.98 & 0.95 & -53.3 & 50.7 \\
\hline & $2 \cdot \mathrm{P}$ & 0.111 & 0.99 & 0.95 & -55.2 & 51.4 \\
\hline \multirow{4}{*}{ Merced River } & No DA & 0.266 & 0.95 & 0.74 & -105.4 & 83.4 \\
\hline & $\mathrm{P}$ & 0.246 & 0.97 & 0.78 & -96.8 & 79.8 \\
\hline & $1 / 2 \cdot P$ & 0.251 & 0.96 & 0.77 & -99.7 & 82.6 \\
\hline & $2 \cdot \mathrm{P}$ & 0.242 & 0.97 & 0.78 & -93.8 & 77 \\
\hline
\end{tabular}

